\title{
STABILOMETRIC ASSESSMENT OF VESTIBULAR FUNCTION IN CHILDREN WITH A MEDIUM OTITIS
}

\author{
Iuliia Lozova, Grigoriy Garyuk, Iryna Redka, Tatiana Pochueva
}

Otitis media is one of the most common infectious diseases of the middle ear in preschool age, but its relationship with vestibular dysfunction remains controversial.

The aim of the study was to carry out a comparative analysis of stabilometric indicators of preschool children with suppurative and non-suppurative otitis media.

Materials and methods: 22 children with suppurative otitis media and 22 children with non-suppurative otitis media at the age from 4 to 7 years were examined by the method of static stabilometry on the device "MPFI Stabilograph 1 ", (LLC "ASTER IT", Kharkov, Ukraine). We took into account the indices of variation and distribution of the center of pressure, spectral and correlation indices of the stabilogram, as well as integral indices of stability (length, velocity and angle of postural oscillations) and the quality of the balance function in two sensory states (open and closed eyes), which were calculated in the software providing StabiliS.

Results. In children with non-suppurative otitis media, the coordinates of the center-of-pressure corresponded (7.04 [2.16-10.09]; -1.14 [-28.90-9.11]), and with suppurative otitis media - (7.04 [3.30-16.16]; -13.72 [(-25.17)-(10.30)]) with closed eyes. The averaged figure of the projection of the center-of-pressure in the test with eyes closed is actually represented by a circle in both groups, but with open eyes it is represented by an ellipse, which, in case of nonsuppurative otitis media, is stretched along the sagittal axis, and in case of suppurative otitis media, along the frontal axis. In both sensory states, children with non-suppurative otitis media were characterized by large ( $p \leq 0.05)$ values of KurtosisX, Length and lower ( $\leq \leq 0.05$ ) values of CCOX, Pup2Sigma compared with non-purulent otitis media.

Conclusions: In preschool age, suppurative otitis media more significantly disrupts the balance function compared with non-suppurative otitis media, which was manifested by a significant decrease in the stability of the main stance and the predictability of oscillatory movements compared with non-suppurative otitis media

Keywords: non-suppurative and suppurative otitis media, stabilometry, balance, vestibular function, preschool age

How to cite:

Lozova, I. Garyuk, G., Redka, I., Pochueva, T. (2021). Stabilometric assessment of vestibular function in children with a medium otitis. ScienceRise: Medical Science, 5 (44), 32-40. doi: http://doi.org/10.15587/2519-4798.2021.241951

(C) The Author(s) 2021

This is an open access article under the Creative Commons CC BY license hydrate

\section{Introduction}

Numerous epidemiological studies around the world [1] show that otitis media is one of the most common infectious diseases of the middle ear in preschool age.

Otitis media is a spectrum of diseases that includes acute otitis media, otitis media with effusion and chronic purulent otitis media. Acute otitis media is manifested by local and systemic symptoms and has a rapid onset. Otitis media with effusion often develops after acute otitis media. Eighty percent of cases of otitis media with effusion are registered before the age of 10 with a maximum of 3 years. The prevalence of otitis media with effusion is about $20 \%$ at the age of 2 years with a decrease in prevalence to $8 \%$ at the age of 8 years [2].

Otitis media affects the middle ear, which is in close proximity to the inner ear, where the labyrinth is located (peripheral part of the vestibular apparatus) [3]. Phylogenetic, functional and anatomical proximity of these structures raise questions about potential morpho- functional changes of the vestibular apparatus due to otitis media.

Histopathological studies [4] revealed a significantly reduced density of hair cells of type I and II in the lateral semicircular canal, sac and uterus; hair cells of type I - in the posterior semicircular canal; dark cells - in the lateral semicircular canal in chronic otitis media. At the same time, no significant changes in hair cell density were detected in serous and serous-purulent otitis media, although there was a tendency to decrease [5]. It has been suggested that a decrease in the amount of vestibular sensory epithelium secondary to otitis media may be the cause of clinical symptoms of imbalance in this disease [4].

A systematic review [6] showed inconsistencies in vestibular function in children with otitis media. Clinical symptoms of autonomic dysfunction, ranging from true dizziness to mild ataxia, were found in $22 \%$ of children with non-purulent otitis media [7]. According to electronystagmography (ENG), the frequency of abnormalities 
is much higher in children with otitis media with effusion $[8,9]$. According to the video pulse test of the head (vHIT) in children with otitis media with dizziness and vertigo has no serious vestibular disorders, but found in this group of patients hidden saccades are regarded as a sign of mild vestibular disorders [10]. Vestibularenhanced myogenic potentiometry (VEMP) with conductive stimulation did not show significant changes in otitis media with effusion, while VEMP with cranial stimulation showed contradictory results: from no change [11] to increased latency [8] and / or a decrease in the amplitude of the evoked potentials [12] in otitis media.

Given that the available vestibular tests in everyday clinical practice (ENG, calorimetric, rotary tests on the armchairs, VEMP) largely depend on the condition of the middle ear and require active cooperation with the child [6], as an alternative, it is proposed to use quantitative methods of static and dynamic posturography (stabilometry) in pediatric practice [6]. The expediency of posturography as a screening method for detecting latent vestibular dysfunction is also due to the fairly common asymptomatic course of otitis media with effusion in children, the only symptoms of which are nonspecific imbalances [13]. The reliability of the results of stabilometric research among children is proven [14].

At the same time, data on motor function and balance function in children with otitis media are contradictory. According to Stott's motor impairment test (STMI) and the advanced motor accuracy test (MAT-R), children with otitis media with effusion were not inferior to the control group [15], while according to the Peabody motor scales (PDMS) and the Bruininks-Oseretsky motor skills test (BOTMP) were the first to show significantly worse results [11]. Most posturographic (stabilometric) studies have a higher mean velocity [16] and amplitude [17] of oscillations in children with otitis media, a greater number of falls during testing [18], a lower mean increase in rotational stimulus of $0.1 \mathrm{~Hz}, 150 \% \mathrm{sec}$ [19]. In contrast, the study [20] found no significant changes in the results of posturography in otitis media [11].

Only isolated works on the long-term effects of otitis media on the vestibular apparatus have been found. In particular, a longitudinal cohort study suggests that pediatric chronic purulent otitis media and recurrent acute otitis media are associated with an increased risk of dizziness in adulthood [21]. A study [22] demonstrated an imbalance in 10-12-year-old children with a history of multiple ear infections and / or tympanostomy tubes before the age of five, according to dynamic computerized posturography with immersion in virtual reality (CDP-IVR), a computerized dynamic test for posturographic stability assessment (SET) and a child balance scale (PBS), which was most pronounced in a tandem rack with eyes closed on an unstable surface.

The aim of the study - a comparative analysis of vestibular function in children with moderate nonpurulent and purulent otitis by stabilometry.

\section{Materials and methods}

The study was conducted on the basis of the children's department of the Municipal Non-Profit Enterprise "City Clinical Hospital No. 30" of the Kharkiv City Council during 2021. The study was agreed with the Ethics Committee of the KCC "City Clinical Hospital No. 30".

44 children aged 4-7 years (mean age $6.06 \pm 0.17$ ) with a uniform distribution in groups with non-purulent and purulent otitis media were examined.

The work was performed in accordance with the Code of Ethics of the World Medical Association (Helsinki Declaration). The work was approved by the Commission on Bioethics of the Kharkiv Medical Academy of Postgraduate Education, protocol No. 3 10/18/2018. Parents of children included in the study gave written informed consent to examine children on a stabilometric platform.

Studies of autonomic-spinal reflexes and balance function were performed in the first 2 days of admission to the hospital by static stabilometry on the device "MPFI stabilograph 1" (LLC "ASTER IT", Kharkiv, Ukraine).

During the study, the child was on a strain gauge platform $(300 \times 300 \mathrm{~mm})$ in an upright position for 1 minute with his eyes closed in a European rack (heels together, socks apart). The movement of the pressure center on the platform area was recorded with a sampling frequency of $13.128 \mathrm{~Hz}$. On the basis of the registered data in the StabiliS software package the following stabilometric indicators were calculated: Length, $\mathrm{mm}$ - length of a trajectory of fluctuations of the center of pressure; AvgSpeed, $\mathrm{mm} / \mathrm{s}$ - average speed of movement of the pressure center; Angle, - the average angle of oscillation of the pressure center; Pup2Sigma, \% - relative number of stabilogram points lying within the double standard deviation; PirsonXY - coefficient of linear correlation between oscillations in the frontal and sagittal planes; RangeX, $\mathrm{mm}$ - amplitude of oscillations of the center of pressure in the frontal plane; RangeY, $\mathrm{mm}$ - the amplitude of oscillations of the center of pressure in the sagittal plane; LengthX, $\mathrm{mm}$ - the length of the trajectory of oscillations of the center of pressure in the frontal plane; LengthY, mm - length of the trajectory of oscillations of the center of pressure in the sagittal plane; MeanX, mmthe average position of the center of pressure in the frontal plane; Mean Y, $\mathrm{mm}$ - average position of the pressure center in the sagittal plane; StdDevX, $m m$ - standard deviation of pressure center oscillations in the frontal plane; StdDevY, mm - standard deviation of pressure center oscillations in the sagittal plane; SkewX, mm - asymmetry of oscillations of the center of pressure in the frontal plane; SkewY, $\mathrm{mm}$ - asymmetry of oscillations of the center of pressure in the sagittal plane; KurtosisX, $m m$ - excess of pressure center oscillations in the frontal plane; KurtosisY, $\mathrm{mm}$ - excess of oscillations of the center of pressure in the sagittal plane; $w A v g F X, H z-$ weighted average frequency of the spectrum of oscillations of the center of pressure in the frontal plane; $w A v g F Y, H z$ - weighted average frequency of the spectrum of oscillations of the pressure center in the sagittal plane; wAvgFXY, $\mathrm{Hz}$ - weighted average frequency of the cross-spectrum of pressure center oscillations in the frontal and sagittal planes; CCOX, $c$ is the offset of the autocorrelation function in the frontal plane to obtain the value of the correlation coefficient less than zero; CCOY, $c$ is the shift of the autocorrelation function in the sagittal plane to obtain the value of the correlation 
coefficient less than zero; $K F R, \%$ - quality of the equilibrium function.

Statistical analysis was performed in the environment of specialized application software STATISTICAv12.5 (StatSoftInc, USA).

The median and interquartile scatter were chosen as measures of the central tendency and distribution of stabilometric indicators due to the inconsistency of the law of normal distribution according to the Shapiro-Wilk criterion. The hypothesis of equality of measures of the central tendency in pairwise comparisons of children with purulent and non-purulent otitis media was tested by the nonparametric U-test Mann-Whitney, and in different sensory states - by the T-test Wilcoxon. Differences at $\mathrm{p} \leq 0.05$ were considered significant.

\section{Results}

The position of the pressure center in children with non-purulent and purulent otitis did not differ statistically significantly $(\mathrm{p}>0.05)$ in any of the planes and was characterized by an absolute shift to the right (only with eyes closed) and back relative to the midline (Table 1). Note that in purulent otitis media, the center of pressure in the sagittal plane is slightly ( $p>0.05$ ) more strongly shifted back relative to the midline compared with nonpurulent otitis (Fig. 1).

Table 1

Coordinates of the position of the pressure center in children with non-purulent and purulent otitis media in different

\begin{tabular}{|c|c|c|c|c|}
\multicolumn{7}{|c}{ sensory states $(\mathrm{Me}[25 \% ; 75 \%])$} \\
\hline Indicators & State & Non-purulent $(\mathrm{n}=22)$ & Purulent $(\mathrm{n}=22)$ & $\mathrm{p}$ \\
\hline \multirow{2}{*}{ MeanX, mm } & CE & $7.04[2.16 ; 10.09]$ & $7.04[3.30 ; 16.16]$ & 0.935 \\
\cline { 2 - 6 } & OE & $-0.04[-5.72 ; 10.43]$ & $-0.72[-5.72 ; 4.47] *$ & 0.549 \\
\hline \multirow{2}{*}{ MeanY, mm } & CE & $-1.14[-28.90 ; 9.11]$ & $-13.72[-25.17 ;-10.30]$ & 0.209 \\
\cline { 2 - 6 } & OE & $-7.81[-35.07 ; 10.08]$ & $-16.35[-23.25 ;-15.05]$ & 0.379 \\
\hline
\end{tabular}

Note: $C E$ - state with closed eyes; $O E$ - state with opened eyes; * - significant differences with the values of indicators in the state with closed eyes

The median values of the asymmetry coefficients (SkewX, SkewY) by module in both groups did not exceed 0.25 , which indicated a relatively symmetrical position of the center of pressure in both planes in children with otitis media (Table 2). There were no statistically significant differences in the symmetry of the main rack in children with purulent and non-purulent otitis media $(\mathrm{p}>0.05)$.
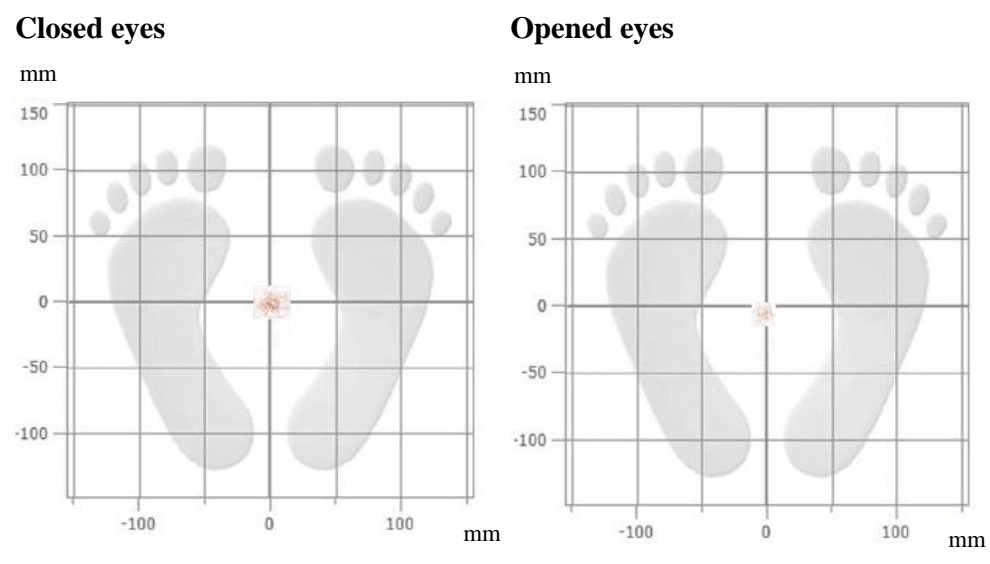

$a$
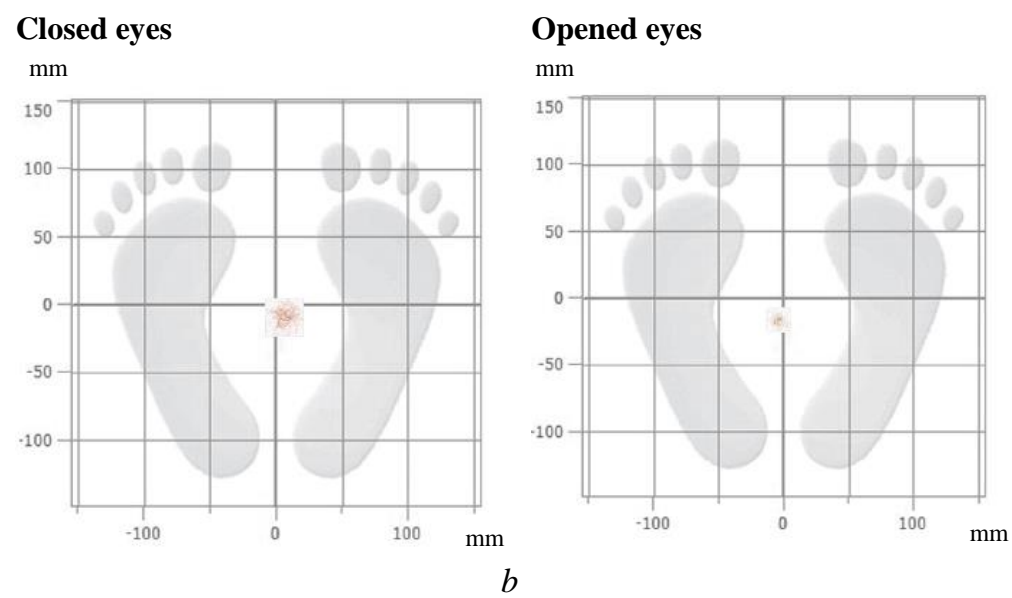

Fig. 1. Example of the position of the pressure center in the coordinate system of the European standard (top) of children with otitis media under different sensory conditions: $a$-non-purulent otitis; $b$ - purulent otitis 
Table 2

Characteristics of the shape of the distribution of oscillations of the pressure center in children with non-purulent and purulent otitis media in different sensory states (Me $[25 \% ; 75 \%])$

\begin{tabular}{|c|c|c|c|c|}
\hline \multirow{2}{*}{ Indicators } & State & Non-purulent otitis $(\mathrm{n}=22)$ & Purulent otitis $(\mathrm{n}=22)$ & $\mathrm{p}$ \\
\hline \multirow{2}{*}{ SkewX } & CE & $-0.03[-0.19 ; 0.15]$ & $-0.03[-0.26 ; 0.10]$ & 0.681 \\
\cline { 2 - 5 } & OE & $0.04[-0.15 ; 0.11]$ & $0.04[-0.21 ; 0.23]$ & 0.681 \\
\hline \multirow{2}{*}{ SkewY } & CE & $0.05[-0.05 ; 0.20]$ & $0.10[-0.06 ; 0.36]$ & 0.897 \\
\cline { 2 - 5 } & OE & $-0.02[-0.23 ; 0.18] *$ & $-0.03[-0.04 ; 0.09]$ & 0.897 \\
\hline \multirow{2}{*}{ KurtosisX } & CE & $\mathbf{2 . 5 8}[2.37 ; 2.84]$ & $\mathbf{2 . 7 4}[2.45 ; 2.92]$ & $\mathbf{0 . 0 2 5}$ \\
\cline { 2 - 5 } & OE & $2.32[2.21 ; 2.57]^{*}$ & $2.42[2.29 ; 2.82]$ & 0.286 \\
\hline \multirow{2}{*}{ KurtosisY } & CE & $2.45[2.21 ; 2.66]$ & $2.58[2.25 ; 2.82]$ & 0.149 \\
\cline { 2 - 5 } & OE & $2.54[2.29 ; 2.61]$ & $2.57[2.55 ; 2.61]$ & 0.177 \\
\hline
\end{tabular}

Note: $C E$ - state with closed eyes; OE - state with opened eyes; * - significant differences with the values of indicators in the state with closed eyes

There are currently two main strategies for maintaining a vertical posture: the ankle strategy, which regulates stability in the posterior-anterior direction, and the hip strategy, which regulates stability in the medio-lateral direction. A physiologically more appropriate strategy to maintain a vertical posture in the conditions of the smallest external perturbations is the ankle strategy, in which the figure of the projection of the center of pressure is represented by an ellipse. According to the results of our study, the average figure of the projection of the pressure center in the sample with closed eyes is actually represented by a circle, both non-purulent (ratio of Y/X axes $=0.99)$ and purulent (Y/X axis ratio $=0.94)$ otitis media, which indicated the lack of dominance of the ankle posture support strategy (Fig. 2,a).

At the same time, in the presence of visual control, the average figure of the projection of the center of pressure is represented by an ellipse, which in nonpurulent otitis elongated along the sagittal axis (ratio of axes $\mathrm{Y} / \mathrm{X}=0.77$ ), and for purulent otitis (ratio of axes $\mathrm{Y} / \mathrm{X}=1.10$ ) - on the frontal axis (Fig. 2, b). Since postural movements around the frontal axis are mainly controlled by antigravity moments created by dorsal/plantar flexion of the ankle joints, and postural movements around the sagittal axis are associated with lateral displacement of body weight due to abduction/reduction of the thigh [23], we could say visual control in children with purulent otitis in providing the main rack begins to dominate the ankle strategy, and in non-purulent otitis the hip strategy remains dominant.

The stability of the main rack was evaluated by the parameters of deviations of the stabilogram points. Statistically significant differences between children with non-purulent and purulent otitis media on the majestic deviations of postural oscillations in both the frontal (RangeX, StdDevX) and sagittal (RangeY, StdDevY) plane were not detected (Table 3). At the same time, children with purulent otitis, compared with non-purulent otitis, were characterized by a statistically lower $(\mathrm{p} \leq 0.05)$ density of stabilogram points within twice the standard deviation (Fig. 2). The value of courtesy in the frontal plane with purulent otitis with closed eyes was statistically higher $(p \leq 0.05)$ than with non-purulent otitis (Table 2). Based on the values of Pup2Sigma and KurtosisX, we could talk about a more pronounced grouping of stabilogram points around the mathematical expectation of the pressure center against the background of higher emissions in the absence of visual control in children with purulent otitis compared with non-purulent otitis.

Indicators of stability of the main rack in children with non-purulent and purulent otitis media in different sensory states Another indicator of the stability of the main posture is the angle of oscillation of the center of pressure, which in children normally does not exceed $12.5^{\circ}$. In our study, no statistically significant differences in this indicator were found between children with nonpurulent and purulent otitis media ( $p>0.05$, Table 3 ).

It was found that children with purulent otitis were characterized by a statistically smaller $(\mathrm{p} \leq 0.05)$ value of the shift of autocorrelation function in the frontal plane, and with open eyes also in the sagittal plane, to obtain a correlation coefficient less than zero compared with children with non-purulent otitis (Table 4). That is, in children with purulent otitis postural fluctuations were more sudden and with greater amplitude. In particular, this is confirmed by the average speed of movement of the pressure center and the total length of the statokinesiogram, which become higher with increasing amplitude and frequency of postural oscillations. Thus, the total length of statokinesiograms was statistically ( $\mathrm{p} \leq 0.05)$ higher than purulent otitis, compared with non-purulent otitis, while AvgSpeed had only a slight tendency $(p>0.05)$ to higher values in children with purulent otitis media, compared with with non-purulent otitis (Tab. 3). AvgSpeed is also a reliable indicator of the effectiveness of postural control and the level of neuromuscular activity required for this [24].

The weighted average frequency of the spectrum of oscillations of the pressure center in the frontal and sagittal planes and the cross-spectrum did not differ statistically significantly in purulent and non-purulent otitis media and was in the low frequency range (about $0.3 \mathrm{~Hz}$ ). This frequency reflects the process of maintaining the center of gravity of the body within the projection of the area of its support and the compensatory restoration of the lost balance of the body as a result of the constituent late reflexes.

Oscillatory movements in the frontal and sagittal planes were independent in both non-purulent and purulent otitis media (Table 4), and the degree of their covariance was similar in both groups $(\mathrm{p}>0.05)$. This is a sign of self-regulation of the main rack in children with otitis media without the involvement of higher central mechanisms of late control. 

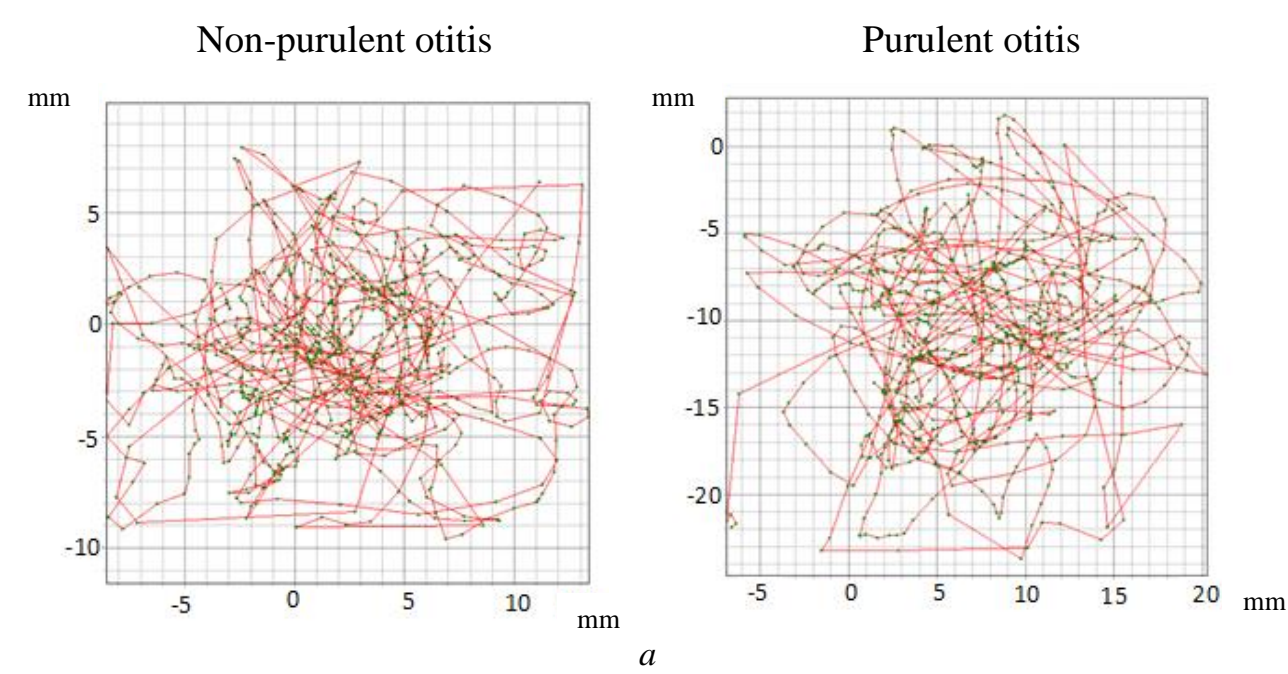

Non-purulent otitis

Purulent otitis
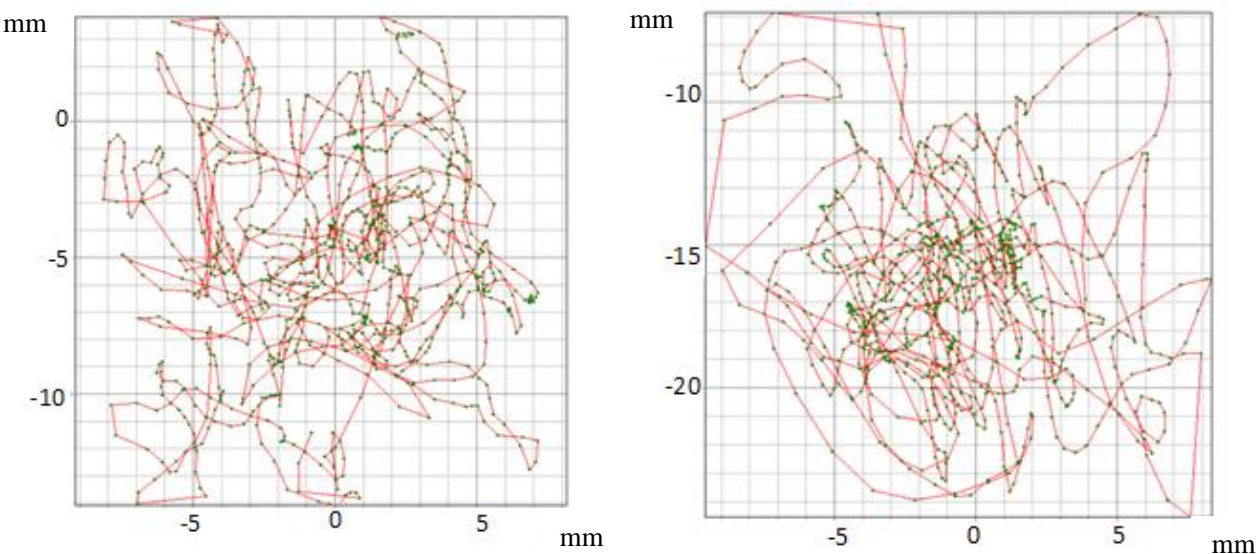

$b$

Fig. 2. Examples of stabilograms of children with non-purulent and purulent otitis media for various sensory states: $a$ - closed eyes; $b$ - open eyes

Table 3

Indicators of the stability of the main rack in children with non-purulent and purulent otitis media in different sensory states (Me $[25 \% ; 75 \%])$

\begin{tabular}{|c|c|c|c|c|}
\hline Indicators & State & Non-purulent otitis $(n=22)$ & Purulent otitis $(n=22)$ & $\mathrm{p}$ \\
\hline \multirow{2}{*}{ Length, mm } & $\mathrm{CE}$ & $\begin{array}{c}1019.00 \\
{[903.20 ; 1447.80]}\end{array}$ & $\begin{array}{c}1445.70 \\
{[1160.70 ; 1543.00]}\end{array}$ & 0.040 \\
\hline & $\mathrm{OE}$ & $\begin{array}{c}\mathbf{7 3 3 . 1 0} \\
{[556.20 ; 899.70] *}\end{array}$ & $\begin{array}{c}\mathbf{8 7 5 . 4 0} \\
{[789.70 ; 987.40] *}\end{array}$ & 0.025 \\
\hline \multirow{2}{*}{ AvgSpeed, mm/s } & $\mathrm{CE}$ & $13.84[12.19 ; 20.48]$ & $19.20[12.76 ; 21.20]$ & 0.354 \\
\hline & $\mathrm{OE}$ & $9.81[7.27 ; 12.08] *$ & $10.69[7.9 ; 11.3] *$ & 0.405 \\
\hline \multirow{2}{*}{ Angle, ${ }^{\circ}$} & $\mathrm{CE}$ & $4.11[-6.02 ; 12.80]$ & $6.01[4.49 ; 10.30]$ & 0.286 \\
\hline & $\mathrm{OE}$ & $3.43[-2.45 ; 13.22]$ & $-0.70[-7.46 ; 8.59]$ & 0.379 \\
\hline \multirow{2}{*}{ Pup2Sigma, $\%$} & $\mathrm{CE}$ & $92.70[91.30 ; 93.40]$ & $91.60[90.00 ; 92.70]$ & 0.028 \\
\hline & $\mathrm{OE}$ & $94.00 *[91.90 ; 95.40]$ & $90.9[90.00 ; 92.70]$ & 0.006 \\
\hline \multirow{2}{*}{ RangeX, mm } & $\mathrm{CE}$ & $27.92[21.87 ; 31.59]$ & $27.12[21.72 ; 39.31]$ & 0.824 \\
\hline & $\mathrm{OE}$ & $25.84[15.28 ; 33.53] *$ & $19.85[17.92 ; 25.45] *$ & 0.330 \\
\hline \multirow{2}{*}{ RangeY, mm } & $\mathrm{CE}$ & $27.61[19.87 ; 33.78]$ & $25.49[23.10 ; 29.05]$ & 0.972 \\
\hline & $\mathrm{OE}$ & $19.93[17.47 ; 25.88] *$ & $21.83[17.9 ; 31.52] *$ & 0.245 \\
\hline \multirow{2}{*}{ StdDevX, mm } & $\mathrm{CE}$ & $5.98[4.49 ; 6.59]$ & $5.00[4.49 ; 7.23]$ & 0.897 \\
\hline & $\mathrm{OE}$ & $5.60[3.19 ; 7.10]$ & $4.31[3.49 ; 5.63] *$ & 0.286 \\
\hline \multirow{2}{*}{ StdDevY, mm } & $\mathrm{CE}$ & $6.06[4.19 ; 7.75]$ & $4.95[4.88 ; 7.40]$ & 0.681 \\
\hline & $\mathrm{OE}$ & $4.05[3.77 ; 5.43]$ & $4.77[3.80 ; 6.59] *$ & 0.614 \\
\hline
\end{tabular}

Note: $C E$ - state with closed eyes; OE - state with opened eyes; *-significant differences with the values of indicators in the state with closed eyes 
Table 4

Spectral-correlation indicators of stabilogram of children with non-purulent and purulent otitis media in different sensory states $(\mathrm{Me}[25 \% ; 75 \%])$

\begin{tabular}{|c|c|c|c|c|}
\hline Indicators & State & Non-purulent otitis $(n=22)$ & Purulent otitis $(n=22)$ & $\mathrm{p}$ \\
\hline \multirow{2}{*}{$\mathrm{CCOX}, \mathrm{s}$} & $\mathrm{CE}$ & $5.67[3.39 ; 12.60]$ & $3.31[1.57 ; 5.91]$ & 0.015 \\
\hline & $\mathrm{OE}$ & $6.90[5.28 ; 11.65]$ & $3.70[2.76 ; 7.32] *$ & 0.022 \\
\hline \multirow{2}{*}{$\mathrm{CCOY}, \mathrm{s}$} & $\mathrm{CE}$ & $6.14[2.76 ; 10.16]$ & $4.00[2.76 ; 7.32]$ & 0.330 \\
\hline & $\mathrm{OE}$ & $6.85[5.28 ; 17.72]$ & $4.41[4.09 ; 6.85]$ & 0.006 \\
\hline \multirow{2}{*}{ wAvgFX, Hz } & $\mathrm{CE}$ & $0.29[0.27 ; 0.33]$ & $0.32[0.27 ; 0.44]$ & 0.103 \\
\hline & $\mathrm{OE}$ & $0.24[0.22 ; 0.32] *$ & $0.24[0.24 ; 0.29] *$ & 0.519 \\
\hline \multirow{2}{*}{ wAvgFY, Hz } & $\mathrm{CE}$ & $0.33[0.29 ; 0.36]$ & $0.34[0.29 ; 0.37]$ & 0.681 \\
\hline & $\mathrm{OE}$ & $0.27[0.25 ; 0.30] *$ & $0.26[0.24 ; 0.28] *$ & 0.209 \\
\hline \multirow{2}{*}{ PirsonXY } & $\mathrm{CE}$ & $0.06[-0.11 ; 0.29]$ & $0.11[0.07 ; 0.15]$ & 0.432 \\
\hline & $\mathrm{OE}$ & $0.05[-0.06 ; 0.22]$ & $-0.02[-0.13 ; 0.17]$ & 0.330 \\
\hline \multirow{2}{*}{ wAvgFXY, Hz } & $\mathrm{CE}$ & $0.30[0.27 ; 0.33]$ & $0.31[0.28 ; 0.33]$ & 0.193 \\
\hline & $\mathrm{OE}$ & $0.24[0.24 ; 0.30] *$ & $0.24[0.24 ; 0.26] *$ & 0.227 \\
\hline
\end{tabular}

Note: CE - state with closed eyes; OE - state with opened eyes; * - significant differences with the values of indicators in the state with closed eyes

No statistically significant $(p>0.05)$ differences in the integrated indicator of postural control were found between children with different forms of otitis media.
However, there was a slight tendency to lower values of the equilibrium quality factor in purulent otitis compared with non-purulent otitis, especially in the absence of visual control (Fig. 3).

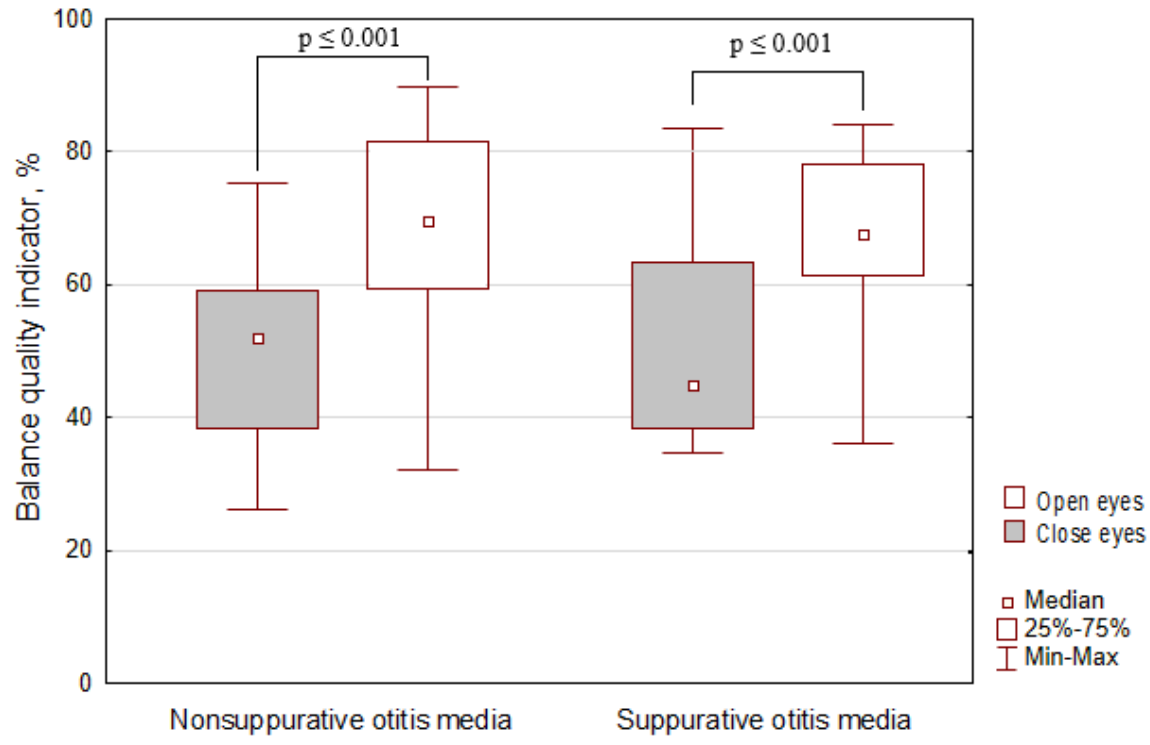

Fig. 3. The quality of the balance function in children with non-purulent and purulent otitis media under different sensory conditions

It was found that the involvement of visual control in maintaining a vertical posture was accompanied by a significant increase in the quality of the equilibrium function (increase in KFR, $\mathrm{p} \leq 0.05$ ) due to increased postmorphological stability, as evidenced by a statistically significant $(\mathrm{p} \leq 0.05)$ decrease in Length, LengthX, LengthY, RangeX, RangeY, AvgSpeed (Table 3), KurtosisX (Table 2), wAvgFX, wAvgFXY, wAvgFY (Table 4) compared to the state of lack of visual information. Despite the similarity of postural effects under the influence of visual afferentation, we found some features depending on the form of otitis media. In the case of purulent otitis media on the background of these changes revealed a significant $(\mathrm{p} \leq 0.05)$ decrease in the values of MeanX (Table 1), StdDevX, StdDevY (Table 3) and an increase $(p \leq 0.05)$ values of $\mathrm{CCOX}$ (Table 4$)$, while in non- purulent otitis media - decrease $(\mathrm{p} \leq 0.05)$ values of SkewY (Table 2) and increase $(p \leq 0.05)$ values of Pup2Sigma ( $\mathrm{p} \leq 0.05$, Table 3$)$. It is likely that visual control of purulent otitis media strengthened the mechanisms of control of postural movements around the sagittal axis, which made them more regular and predictable and shifted the coordinates of the pressure center closer to the absolute center in the medio-lateral direction, creating conditions for more effective implementation. In children with non-purulent otitis media, visual control contributed to the strengthening of the symmetry of oscillations in the anterior-posterior direction.

\section{Discussion}

Despite the signs of latent vestibular dysfunction, which are observed in children with otitis media, they 
often go unnoticed in clinical practice due to the asymptomatic course of otitis media with effusion [13, 25], atypical manifestation of vestibular symptoms and limited communicative abilities of children in relation to their description [26, 27]. At the same time, the vestibular system is important for the normal psychomotor, cognitive and socio-emotional development of the child $[13,28]$. That is why clinicians should be aware of the possibility of adverse effects of otitis media on vestibular function [16]. In order to expand modern ideas on this issue, we conducted a study aimed at comparative analysis of stabilometric parameters in preschool children with different forms (non-purulent and purulent) of otitis media.

The main result of our study is to identify the fact of a statistically significant decrease in the stability of the main posture and predictability of oscillatory movements in preschool children with purulent otitis media compared with non-purulent otitis media, regardless of visual control. Since the maintenance of vertical posture in conditions of relative rest is provided mainly by vestibulospinal constitutive late reflexes, the data we found may indicate the presence of changes in vestibular function.

If we consider non-purulent and purulent average as clinical models of different degrees of pathological process, the obtained data by us are consistent with the results of histopathological studies on a more pronounced violation of the sensory epithelium of the vestibular analyzer with the progression of the pathological process in the inner ear [4].

Our data are also consistent with the available hypotheses about the pathogenesis of vestibular disorders in otitis media. Thus, according to one theory, the change in pressure inside the middle ear affects the flow of fluid inside the inner ear, as a consequence, affecting the balance function [9]. According to another hypothesis, toxins and / or mediators of inflammation from the middle ear can enter the inner ear through natural membranous barriers and cause inflammation of hair cells or damage to the blood-perilymph barrier, changing the density gradient between perilymph and endolymph [29]. The following hypothesis assigns a leading role to the altered electrolyte composition of the middle ear, which enters the inner ear due to the transfer of ions through semipermeable membranes, changing the chemical composition of perilymph and endolymph and, consequently, affecting the ion channels of kinocilia and stereocilia [7, 18]. It is obvious that in purulent otitis media the concentration of toxins and mediators of inflammation, as well as changes in the electrolyte composition in the middle ear is greater than in non-purulent otitis media, which probably led to a more pronounced imbalance.

We also found that the inclusion of visual afferents contributes to the predominance of the ankle strategy to provide the main posture in children with purulent otitis media, while in non-purulent otitis media the hip strategy remains dominant. Since the ankle strategy is physiologically more appropriate, and in healthy children under 12 years of age in postural con- trol plays a greater role proprioceptive-vestibular integration than vestibular-visual integration [30], we can assume that in purulent otitis more pronounced changes in the vestibular visual integration against the background of increasing "physiological price" of postural control.

Due to the lack of generally accepted standards of stabilometric indicators and the need for clinical evaluation of the detected dysfunction in children with purulent otitis media, we consider it appropriate to conduct further comparative analysis of stabilometric indicators of otitis media with those of healthy peers.

Study limitations no assessment of the role of proprioceptive sensitivity to maintain postural balance, and considered only the role of visual and vestibular sensory afferents. At the same time, this was not a special subject of our study and may be a prospect for further research. Another limitation is the wide age range of the studied groups, however, the age representation of children is comparable in groups with non-purulent and purulent otitis.

Prospects for further research. In order to clarify the state of vestibular function in otitis media in children, it is advisable to compare their stabilometric indicators with those in almost healthy children, as there are currently no generally accepted clinical standards for stabilometric indicators.

\section{Conclusions}

In preschool age, purulent otitis media significantly impairs the function of balance compared with nonpurulent otitis media, as evidenced by significantly higher values of Kurtosis $X$, Length $(p \leq 0.05)$ and lower values of Pup2Sigma, CCOX, CCOY $(\mathrm{p} \leq 0.05)$. The inclusion of visual afferents facilitates the maintenance of vertical balance (decrease in the values of Length, LengthX, LengthY, RangeX, RangeY, AvgSpeed, wAvgFX, wAvgFXY, wAvgFY, $\mathrm{p} \leq 0.05$ ) in preschool children with otitis media, but not in different ways: due to a decrease in the amplitude of postural movements (decrease in the values of SkewY, Pup2Sigma, $\mathrm{p} \leq 0.05$ ), and in purulent otitis - due to a decrease in their variability (decrease in the values of MeanX, StdDevX, StdDevY, $p \leq 0.05$ ). In the presence of visual control in children with purulent otitis in the provision of the main posture begins to dominate the ankle strategy (ratio of axes I / X=1.10 in the state with open eyes), and in non-purulent otitis the dominant hip strategy (ratio of axes $\mathrm{Y} / \mathrm{X}=0.77$ in a state with open eyes).

Stabilometry can be recommended as a screening method to detect latent vestibular disorders in preschool children with otitis media.

\section{Conflict of interests} interest.

The authors declare that they have no conflicts of

Financing

The study was performed without financial support. 


\section{References}

1. DeAntonio, R., Yarzabal, J.-P., Cruz, J. P., Schmidt, J. E., Kleijnen, J. (2016). Epidemiology of otitis media in children from developing countries: A systematic review. International Journal of Pediatric Otorhinolaryngology, 85, 65-74. doi: http://doi.org/10.1016/ j.ijporl.2016.03.032

2. Zernotti, M. E., Pawankar, R., Ansotegui, I., Badellino, H., Croce, J. S., Hossny, E. et. al. (2017). Otitis media with effusion and atopy: is there a causal relationship? World Allergy Organization Journal, 10, 37. doi: http://doi.org/10.1186/s40413-017-0168-x

3. Said, E. A.-F. (2014). Vestibular assessment in children with sensorineural hearing loss using both electronystagmography and vestibular-evoked myogenic potential. The Egyptian Journal of Otolaryngology, 30 (1), 43-52. doi: http://doi.org/10.4103/ $1012-5574.127203$

4. Da Costa Monsanto, R., Erdil, M., Pauna, H. F., Kwon, G., Schachern, P. A., Tsuprun, V. et. al. (2016). Pathologic Changes of the Peripheral Vestibular System Secondary to Chronic Otitis Media. Otolaryngology-Head and Neck Surgery, 155 (3), $494-500$. doi: http://doi.org/10.1177/0194599816646359

5. Monsanto, R. da C., Schachern, P., Paparella, M. M., Cureoglu, S., Penido, N. de O. (2017). Progression of changes in the sensorial elements of the cochlear and peripheral vestibular systems: The otitis media continuum. Hearing Research, 351, 2-10. doi: http://doi.org/10.1016/j.heares.2017.05.003

6. Monsanto, R. da C., Kasemodel, A. L. P., Tomaz, A., Paparella, M. M., Penido, N. de O. (2018). Current evidence of peripheral vestibular symptoms secondary to otitis media. Annals of Medicine, 50 (5), 391-401. doi: http://doi.org/10.1080/07853890.2018.1470665

7. Grace, A. R. H., Pfleiderer, A. G. (1990). Dysequilibrium and otitis media with effusion: What is the association? The Journal of Laryngology \& Otology, 104 (9), 682-684. doi: http://doi.org/10.1017/s0022215100113611

8. Kolkaila, E. A., Emara, A. A., Gabr, T. A. (2015). Vestibular evaluation in children with otitis media with effusion. The Journal of Laryngology \& Otology, 129 (4), 326-336. doi: http://doi.org/10.1017/s0022215115000535

9. Myers, E. N., Koyuncu, M., Saka, M. M., Tanyeri, Y., Şleşlen, T., ünal, R. et. al. (1999). Effects of Otitis Media with Effusion on the Vestibular System in Children. Otolaryngology-Head and Neck Surgery, 120 (1), 117-121. doi: http://doi.org/10.1016/s01945998(99)70381-5

10. Tozar, M., Cömert, E., Şencan, Z., Şimşek, G., Muluk, N. B., Kılıç, R. (2020). Video head impulse test in children with otitis media with effusion and dizziness. International Journal of Pediatric Otorhinolaryngology, 129, 109783. doi: http://doi.org/10.1016/ j.ijporl.2019.109783

11. Said, E. A., Ahmed, M. K., Mohamed, E. S. (2015). Role of vestibular testing in deciding treatment strategies for children with otitis media with effusion. Egyptian Journal of Ear, Nose, Throat and Allied Sciences, 16 (2), 151-159. doi: http://doi.org/10.1016/ j.ejenta.2015.05.003

12. Wang, M.-C., Lee, G.-S. (2007). Vestibular evoked myogenic potentials in middle ear effusion. Acta Oto-Laryngologica, 127 (7), 700-704. doi: http://doi.org/10.1080/00016480601002070

13. Robb, P., Williamson, I.; Watkinson, J. C., Clarke, R. W. (Eds.) (2018). Otitis media with effusion. Scott Brown's otolaryngology head \& neck surgery. Boca Raton: CRC Press, 115-135. doi: http://doi.org/10.1201/9780203731017-13

14. Barozzi, S., Socci, M., Soi, D., Di Berardino, F., Fabio, G., Forti, S. et. al. (2014). Reliability of postural control measures in children and young adolescents. European Archives of Oto-Rhino-Laryngology, 271 (7), 2069-2077. doi: http://doi.org/10.1007/s00405014-2930-9

15. Von, T., Deitz, J. C., McLaughlin, J., DeButts, S., Richardson, M. (1988). The Effects of Chronic Otitis Media on Motor Performance in 5- and 6-Year-Old Children. The American Journal of Occupational Therapy, 42 (7), 421-426. doi: http://doi.org/10.5014/ ajot.42.7.421

16. Pazdro-Zastawny, K., Pośpiech, L., Zatoński, T. (2018). Long-term evaluation of the effect of middle ear effusion on the vestibular system in children. International Journal of Pediatric Otorhinolaryngology, 109, 13-16. doi: http://doi.org/10.1016/j.ijporl.2018.03.015

17. Jones, N. S., Prichard, A. J. N., Radomskij, P., Snashall, S. E. (1990). Imbalance and Chronic Secretory Otitis Media in Children: Effect of Myringotomy and Insertion of Ventilation Tubes on Body Sway. Annals of Otology, Rhinology \& Laryngology, 99 (6), 477-481. doi: http://doi.org/10.1177/000348949009900612

18. Casselbrant, M. L., Villardo, R. J. M., Mandel, E. M. (2008). Balance and otitis media with effusion. International Journal of Audiology, 47 (9), 584-589. doi: http://doi.org/10.1080/14992020802331230

19. Casselbrant, M. L., Furman, J. M., Mandel, E. M., Fall, P. A., Kurs-Lasky, M., Rockette, H. E. (2000). Past History of Otitis Media and Balance in Four-Year-Old Children. The Laryngoscope, 110 (5), 773-778. doi: http://doi.org/10.1097/00005537200005000-00007

20. Ben-David, J., Podoshin, L., Fradis, M., Faraggi, D. (1993). Is the Vestibular System Affected by Middle Ear Effusion? Otolaryngology-Head and Neck Surgery, 109 (3), 421-426. doi: http://doi.org/10.1177/019459989310900306

21. Aarhus, L., Tambs, K., Hoffman, H. J., Engdahl, B. (2015). Childhood otitis media is associated with dizziness in adulthood: the HUNT cohort study. European Archives of Oto-Rhino-Laryngology, 273 (8), $2047-2054$. doi: http://doi.org/10.1007/s00405-015-3764-9

22. Sabir, O. A., Johnson, E. G., Hafiz, A. E., Nelson, R. N., Hudlikar, M., Sheth, I., Daher, N. S. (2021). Chronic Effects of Pediatric Ear Infections on Postural Stability. International Journal of Pediatrics, 2021, 1-6. doi: http://doi.org/10.1155/2021/6688991

23. Wang, Z., Newell, K. M. (2014). Inter-foot coordination dynamics of quiet standing postures. Neuroscience \& Biobehavioral Reviews, 47, 194-202. doi: http://doi.org/10.1016/j.neubiorev.2014.08.007

24. Paillard, T., Noé, F. (2015). Techniques and Methods for Testing the Postural Function in Healthy and Pathological Subjects. BioMed Research International, 2015, 1-15. doi: http://doi.org/10.1155/2015/891390

25. Schilder, A. G. M., Chonmaitree, T., Cripps, A. W., Rosenfeld, R. M., Casselbrant, M. L., Haggard, M. P., Venekamp, R. P. (2016). Otitis media. Nature Reviews Disease Primers, 2 (1). doi: http://doi.org/10.1038/nrdp.2016.63

26. Rine, R. (2018). Vestibular Rehabilitation for Children. Seminars in Hearing, 39 (3), 334-344. doi: http://doi.org/10.1055/ s-0038-1666822

27. Rehagen, S. K., Valente, M., Lieu, J. E. C. (2020). Vestibular Screening in Pediatric Patients with Otitis Media. Journal of the American Academy of Audiology, 31 (3), 209-216. doi: http://doi.org/10.3766/jaaa.18101

28. Lacroix, E., Edwards, M. G., De Volder, A., Noël, M.-P., Rombaux, P., Deggouj, N. (2020). Neuropsychological profiles of children with vestibular loss. Journal of Vestibular Research, 30 (1), 25-33. doi: http://doi.org/10.3233/ves-200689 
29. Choi, J. W., Han, K., Nahm, H., Shin, J. E., Kim, C.-H. (2019). Direction-Changing Positional Nystagmus in Acute Otitis Media Complicated by Serous Labyrinthitis: New Insights into Positional Nystagmus. Otology \& Neurotology, 40 (4), e393-e398. doi: http://doi.org/10.1097/mao.0000000000002104

30. Casselbrant, M. L., Villardo, R. J. M., Mandel, E. M. (2008). Balance and otitis media with effusion. International Journal of Audiology, 47 (9), 584-589. doi: http://doi.org/10.1080/14992020802331230

Received date 03.08.2021

Accepted date 07.09.2021

Published date 30.09.2021

Iuliia Lozova*, Postgraduate Student, Department of Otorhinolaryngology and Pediatric Otolaryngology, Kharkiv Medical Academy of Postgraduate Education, Amosova str., 58, Kharkiv, Ukraine, 61176, Head of Department, Children's Otolaryngology Department, Kharkiv City Clinical Hospital No. 30

Hudanova str., 5/7, Kharkiv, Ukraine, 61024

Grigoriy Garyuk, Doctor of Medical Sciences, Professor, Head of Department, Honored Worker of Science and Technology of Ukraine, Department of Otorhinolaryngology and Pediatric Otolaryngology, Kharkiv Medical Academy of Postgraduate Education, Amosova str., 58, Kharkiv, Ukraine, 61176

Iryna Redka, PhD, Assosiate Professor, Department of Hygiene and Social Medicine, V. N. Karazin Kharkiv National University, Svobody sq., 4, Kharkiv, Ukraine, 61022

Tatiana Pochueva, Doctor of Medical Sciences, Professor, Department of Otorhinolaryngology and Pediatric Otolaryngology, Kharkiv Medical Academy of Postgraduate Education, Amosova str., 58, Kharkiv, Ukraine, 61176

*Corresponding author: Iuliia Lozova, e-mail: ukrlor@gmail.com 